\title{
Health knowledge and perception of risks among Chinese smokers and non-smokers: findings from the Wave 1 ITC China Survey
}

\author{
Jilan Yang, ${ }^{1}$ David Hammond, ${ }^{1,2}$ Pete Driezen, ${ }^{2}$ Geoffrey T Fong, ${ }^{3,4}$ Yuan Jiang ${ }^{5}$
}

${ }^{1}$ Department of Health Studies and Gerontology, University of Waterloo, Waterloo, Canada ${ }^{2}$ Propel Centre for Population Health Impact, University of Waterloo, Waterloo, Canada ${ }^{3}$ Department of Psychology, University of Waterloo, Waterloo, Canada ${ }^{4}$ Ontario Institute for Cancer Research, Canada ${ }^{5}$ National Tobacco Control Office, Chinese Center for Disease Control and Prevention, Beijing, China

\section{Correspondence to} Dr David Hammond, Department of Health Studies and Gerontology, University of Waterloo, 200 University Ave West, Waterloo, Canada N2L 3G1; dhammond@uwaterloo.ca

Received 24 February 2009 Accepted 29 September 2009

\section{(2) UNLOCKAD}

This paper is freely available online under the BMJ Journals unlocked scheme, see http:// tobaccocontrol.bmi.com/site/ about/unlocked.xhtml

\section{ABSTRACT \\ Background Awareness of health risks of smoking is strongly associated with smoking behaviour. However, there are no population-based studies of smoking-related health knowledge in China. \\ Objective The aim of current study was to use a population-based sample from the International Tobacco Control China Wave 1 survey to examine variations between current, former and never smokers' health knowledge about smoking and the impact of health knowledge awareness on smokers' intention to quit.}

Methods A face-to-face interview was conducted with 5986 adult smokers and non-smokers from six cities in China. Respondents were asked whether they believed smoking causes heart disease, stroke, impotence, lung cancer, emphysema, stained teeth, premature ageing in smokers and lung cancer in non-smokers. Current smokers were also asked additional questions on how smoking affects their current and future health as well as whether they had plans to quit smoking and if they believe they would have health benefit from quitting.

Findings The overall awareness of health risks of smoking in China was low compared to developed countries. Current smokers in China were less likely than non-smokers and former smokers to acknowledge the consequences of smoking. Current smokers who were more aware of the health consequences of smoking were more likely to intend to quit smoking.

Conclusion These findings highlight the need to increase awareness about the health effects of smoking in China, particularly among current smokers to increase quitting.

\section{INTRODUCTION}

At present, approximately 1.3 billion people smoke and more than 5 million people die globally from smoking each year. ${ }^{1}$ In contrast to the decline in smoking prevalence among high income countries, tobacco use continues to rise in low and middleincome countries. ${ }^{1}$ As a consequence, global tobacco consumption is still increasing and smoking attributable death is projected to rise beyond eight million deaths per year by 2030 .

Approximately one-third of the world's smokers-350 million - live in China. Recent estimates suggest that more than a third of Chinese over the age of 15 are smokers, with dramatic differences between genders: approximately $66 \%$ of Chinese males smoke compared to only $3 \%$ of Chinese females. $^{2}$ In addition, more than $70 \%$ of all Chinese, or over 600 million Chinese people, are regularly exposed to secondhand smoke. ${ }^{3}$ Given that smoking is a major contributor to chronic obstructive pulmonary disease and lung cancer, both of which are leading causes of death in China, the health burden from tobacco use in China is daunting: tobacco use is estimated to have caused approximately one million premature deaths in 2000 and the death toll is projected to double by 2020 if the present smoking trends continue. ${ }^{4} 5$ Overall, up to 100 million Chinese smokers currently under 30 years old are projected to die from smoking. ${ }^{1}$ Thus, the success of global tobacco control relies to a large extent upon reductions of smoking in China.

Tobacco control policies have emerged more slowly in China than in many other countries in the region. Until October 2008, Chinese cigarette packages carried obscure health warnings printed on the side of the pack with the vague text-only message that, 'Smoking may harm your health'. Although tobacco advertisements have been banned from mass media, such as TV, radio and newspapers, tobacco companies have successfully used sponsorships and promotions to maintain a visible marketing presence. ${ }^{6}$ To date, China does not have a national law to restrict smoking in workplaces and other indoor public venues, while smoking remains common in healthcare facilities and educational facilities. ${ }^{7}$ In short, both the state of existing tobacco control regulations in China and their enforcement remain at an early stage.

A primary focus of tobacco control is to raise awareness of the health risks of smoking, and this may be particularly true in low and middle-income countries, most of which have not engaged in campaigns to educate the public on the hazards of smoking. Although increases in perceptions of risk are not always sufficient to reduce smoking on their own, increases in health knowledge are strongly associated with reductions in smoking initiation, increases in cessation behaviour and long-term abstinence from smoking. ${ }^{89}$ Increases in health knowledge also provide support for other tobacco control measures, such as increases in taxation and more comprehensive workplace smoking restrictions.

In China, as in many other low and middleincome countries, there is little published information about the level of health knowledge about smoking. ${ }^{1}$ A study conducted among industrial workers in Shanghai two decades ago found that only $53 \%$ of smokers and $76 \%$ of non-smokers were generally aware that smoking is harmful to health, and only $51 \%$ of smokers reported that smoking 
causes lung cancer. ${ }^{10}$ A more recent study among Chinese physicians found that a majority knew that smoking causes lung cancer and other chronic obstructive pulmonary disease, but only two-thirds (67\%) knew that smoking could cause heart disease. ${ }^{11}$ These findings are particularly troubling given that smoking is a primary risk factor for heart disease, another leading cause of premature death in China. ${ }^{12}$ Similar gaps in knowledge have been identified in other studies with healthcare providers. ${ }^{13} 14$ However, to our knowledge, there are no population-based studies of smoking-related health knowledge in China.

The purpose of this study was to examine beliefs about the health risks of smoking in China among never, former and current smokers. The study also examined sociodemographic differences in the levels of health beliefs, as well as associations with intentions to quit smoking among current smokers.

\section{METHODS}

The ITC China Survey is a prospective cohort survey of 800 adult smokers and 200 adult non-smokers in each of six cities in China: Beijing, Shanghai, Guangzhou, Shenyang, Changsha and Yinchuan. The six cities in the ITC China Survey were selected based on geographical representativeness and levels of economic development. In each city, the ITC China Survey employed a multistage cluster sampling design. Ten street districts (Jie Dao) were randomly selected from each city with probability of selection proportional to the population size of the Jie Dao. Within each of these Jie Dao, two residential blocks (Ju Wei Hui) were selected, again with probability proportional to the population size of the Ju Wei Hui. Within each selected Ju Wei Hui, a complete list of addresses of the dwelling units (households) was first compiled, and then a sample of 300 households were drawn from the list by simple random sampling without replacement in each city.

In the enumeration process, information on age, gender and smoking status for all adults living in these 300 households was collected. The enumerated 300 households were then randomly ordered, and adult smokers and non-smokers were subsequently approached following the randomised order until 40 adult smokers and 10 adult non-smokers were surveyed. Because of low smoking prevalence among women, one male smoker and one female smoker from every selected household were surveyed whenever possible to increase the sample size for female smokers. At most, one non-smoker was interviewed per household. Where there was more than one person in a sampling category to choose from in a household, the next birthday method was used to select the individual to be interviewed. Up to four visits to a household were made in order to interview the target person(s) within that household. All surveys were conducted 'face-to-face.'

The findings reported here are from Wave 1 of the ITC China Survey, which was conducted between April and August 2006. The Wave 1 cooperation rates, calculated as ratio of completed interviews among the total number of successfully contacts in the initial sample recruitment plan, ranged from $80.0 \%$ in Beijing and Guangzhou to $95.0 \%$ in Changsha. The response rate, calculated as the ratio of completed interviews among the total number of respondents selected in the initial sample plan, ranged from $39.4 \%$ in Yinchuan to $66.0 \%$ in Guangzhou. Additional information on survey rates is available in the ITC China technical report. ${ }^{15}$

The enumerators and survey interviewers were organised and trained by China Center for Disease Control (CDC) staff in each city, with supervision from the ITC China Project team at the
China National CDC. Several quality control procedures were put in place, including making MP3 recordings for each of the 800 smoker interviews in each of the six cities, with subsequent random monitoring. All materials and procedures used in the ITC China Survey were reviewed and cleared for ethics by the research ethics board at the University of Waterloo and by the institutional review board at China National Center for Disease Control and Prevention. Additional detail on the ITC China survey is described elsewhere. ${ }^{15}$

\section{Measures}

\section{Demographic variables}

Age, gender, education and income were recorded in the survey. Age was categorised into four groups as '18-24; 25-39; 40-54; $55+$ '. Education level was categorised into 'low (no education and elementary school); middle (junior high school and high school); high (college and higher)'. Household monthly income was classified as '1000 yuan and under; 1001-5000 yuan; 5001 yuan and above'.

\section{Smoking status classification}

Respondents who had smoked at least 100 cigarettes in their lifetime and who were smoking at the time of the survey were classified as current smokers. Smokers who smoked at least 100 cigarettes in their lifetime but who were not smoking at the time of the survey were classified as former smokers. Those respondents who had smoked fewer than 100 cigarettes were classified as never smokers.

For current smokers, intention to quit was measured by 'Are you planning to quit in the next month, 6 months, beyond 6 months, or not at all?' and categorised into two groups; $1=$ 'In the next month/ 6 months/beyond 6 month' and $0=$ 'not at all'.

\section{Health knowledge of smoking}

Respondents were asked whether they believed smoking causes heart disease, stroke, impotence, lung cancer, emphysema, stained teeth, premature ageing in smokers and lung cancer in non-smokers. Responses were coded as $0=$ 'no/don't know' versus $1=$ 'yes.' A health knowledge scale was created by summing the number of 'yes' responses across the 8 diseases/health effects (range $=0$ to 8 ).

\section{Other health beliefs}

For current smokers, other health beliefs were measured by asking if respondents agreed with the following statements: (1) 'Every cigarette you take damages your health'; (2) 'Tobacco is addictive'; and (3) 'Your cigarette smoke is dangerous to nonsmokers'. Responses were given on a five-point Likert scale where $1=$ 'strongly disagree' and 5='strongly agree'. Current smokers were also asked three additional questions: (1) 'How much do you think you would benefit from health and other gains if you were to quit smoking permanently in the next 6 months'; (2) 'To what extent, if at all, has smoking damaged your health'; and (3) 'How worried are you, if at all, that smoking will damage your health in the future?' Response categories were 'not at all', 'a little', 'very much' and 'don't know'.

\section{ANALYSIS}

All statistical analyses were performed using SAS version 9.1 (SAS Institute Inc). Analyses were weighted on sex and age within each city to account for the sampling design of the ITC China Survey. $\chi^{2}$ Tests were conducted to examine bivariate differences with smoking status. All odds ratios presented for 
Table 1 Sample characteristics $(n=5986)$

\begin{tabular}{|c|c|c|c|c|c|c|c|c|c|}
\hline & & \multicolumn{2}{|c|}{ Never smokers } & \multicolumn{2}{|c|}{ Former smokers } & \multicolumn{2}{|c|}{ Current smokers } & \multicolumn{2}{|c|}{ Overall } \\
\hline & & $\%$ & $\mathbf{n}$ & $\%$ & $n$ & $\%$ & $\mathbf{n}$ & $\%$ & $n$ \\
\hline \multirow[t]{2}{*}{ Sex } & Male & 32.4 & 348 & 90.8 & 168 & 95.1 & 4494 & 83.7 & 5010 \\
\hline & Female & 67.6 & 726 & 9.2 & 17 & 4.9 & 233 & 16.3 & 976 \\
\hline \multirow[t]{4}{*}{ Age } & $18-24$ & 5.0 & 54 & 0.0 & 0 & 1.4 & 66 & 2.0 & 120 \\
\hline & $25-39$ & 22.4 & 240 & 8.7 & 16 & 17.6 & 831 & 18.2 & 1087 \\
\hline & $40-54$ & 42.3 & 454 & 27.6 & 51 & 49.4 & 2335 & 47.4 & 2840 \\
\hline & $55+$ & 30.4 & 326 & 63.8 & 118 & 31.6 & 1495 & 32.4 & 1939 \\
\hline \multirow[t]{4}{*}{ Income } & Low & 18.5 & 199 & 18.9 & 35 & 19.6 & 925 & 19.4 & 1159 \\
\hline & Moderate & 71.1 & 764 & 71.9 & 133 & 69.6 & 3291 & 70.0 & 4188 \\
\hline & High & 2.9 & 31 & 2.2 & 4 & 3.7 & 173 & 3.5 & 208 \\
\hline & No answer & 7.4 & 80 & 7.0 & 13 & 7.2 & 338 & 7.2 & 431 \\
\hline \multirow[t]{3}{*}{ Education } & Low & 10.3 & 111 & 20.5 & 38 & 13.1 & 620 & 12.8 & 769 \\
\hline & Moderate & 60.1 & 645 & 55.1 & 102 & 65.5 & 3098 & 64.2 & 3845 \\
\hline & High & 29.6 & 318 & 24.3 & 45 & 21.3 & 1009 & 22.9 & 1372 \\
\hline \multirow[t]{4}{*}{ Cigarette smoked/day } & $0-10$ & & & & & 34.9 & 1639 & & \\
\hline & $11-20$ & & & & & 49.0 & 2304 & & \\
\hline & $21-30$ & & & & & 8.6 & 404 & & \\
\hline & $31+$ & & & & & 7.5 & 354 & & \\
\hline
\end{tabular}

the logistic regression model were adjusted for gender, age, income and education.

\section{RESULTS}

\section{Sample characteristics}

As shown in table 1, more than $90 \%$ of current and former smokers surveyed were male. The majority of respondents $(65.6 \%)$ were $25-54$ years old and $83.9 \%$ of current smokers smoked fewer than 20 cigarettes per day.

\section{Health knowledge overall}

Table 2 shows the extent to which respondents agreed that smoking was indeed a cause of each of the eight health effects. Overall, respondents were most likely to agree that smoking causes stained teeth and lung cancer, followed by emphysema and lung cancer from secondhand smoke. Only about $40 \%$ of respondents agreed that smoking causes coronary heart disease (CHD), while only one-fifth agreed that smoking causes stroke and impotence. Approximately $7 \%$ of respondents agreed that smoking caused all eight of the health effects.

Significant differences were observed in health knowledge between current, former and never smokers. Overall, current smokers agreed with fewer health effects (mean $=3.82$ out of 8 ) compared to former smokers (mean $=5.5 ; \mathrm{p}<0.001)$ and never smokers (mean $=5.5 ; \mathrm{p}<0.001)$. For individual health effects, only $68 \%$ of current smokers agreed that smoking causes lung cancer in smokers, compared to more than $90 \%$ of former and never smokers. In addition, only $36 \%$ of current smokers agreed that smoking causes $\mathrm{CHD}$, compared to more than half of former and never smokers.

Logistic regression models were conducted to examine differences between current, former and never smokers for each health effect, adjusting for sex, age, income and education. Current smokers were significantly less likely than never and former smokers to agree with each of the eight health effects $(p<0.001$ in all cases). No significant differences were observed between former and never smokers for any of the eight health effects. Linear regression models were also conducted to examine potential differences in the overall health knowledge scale, adjusting for age, sex, income and education. The pattern was the same as for the individual health effects: current smokers had significantly lower health knowledge scores than never smokers (mean difference $=-1.5 ; \mathrm{p}<0.001$ ) and former smokers (mean difference $=-1.8 ; \mathrm{p}<0.001$ ), with no difference between never and former smokers.

Additional analyses were conducted to examine the association between sociodemographic factors and measures of health knowledge among current smokers. Higher levels of health knowledge were observed among: older smokers (40-54 years vs 25-39 years, $\mathrm{OR}=1.78 \mathrm{p}=0.018$ ); more educated smokers (high vs. low, $\mathrm{OR}=2.56 \mathrm{p}=0.007)$; and smokers with lower daily cigarette consumption $(21-30$ vs $0-10 \mathrm{CPD}, \mathrm{OR}=0.42 \mathrm{p}=0.008$; $11-20$ vs $0-10 \mathrm{CPD}, \mathrm{OR}=0.72 \mathrm{p}=0.02$ ). No other significant

Table 2 Agreement of each knowledge of health effect by smoking status $(n=5986)$

\begin{tabular}{|c|c|c|c|c|c|c|c|c|}
\hline \multirow[b]{2}{*}{ Knowledge of health effect } & \multicolumn{2}{|c|}{$\begin{array}{l}\text { Never smokers } \\
(n=1074)\end{array}$} & \multicolumn{2}{|c|}{$\begin{array}{l}\text { Former smokers } \\
(n=185)\end{array}$} & \multicolumn{2}{|c|}{$\begin{array}{l}\text { Current smokers } \\
(\mathrm{n}=4732)\end{array}$} & \multicolumn{2}{|l|}{ Overall } \\
\hline & $\%$ & $\mathbf{n}$ & $\%$ & $\mathbf{n}$ & $\%$ & $\mathbf{n}$ & $\%$ & $\mathbf{n}$ \\
\hline Smoking causes stained teeth & 95.0 & 1026 & 96.1 & 178 & 85.0 & 4024 & $87.2^{*}$ & 5288 \\
\hline Smoking causes lung cancer in smokers & 91.8 & 996 & 90.2 & 162 & 68.1 & 3,244 & $73.0^{*}$ & 4402 \\
\hline Smoking causes emphysema & 86.9 & 935 & 87.2 & 158 & 59.4 & 2858 & $65.2^{*}$ & 3951 \\
\hline Secondhand smoke causes lung cancer in non-smokers & 83.2 & 889 & 76.9 & 135 & 53.2 & 2,531 & $59.2^{*}$ & 3555 \\
\hline Smoking causes premature ageing & 76.0 & 821 & 74.2 & 131 & 47.3 & 2,268 & $53.3^{*}$ & 3220 \\
\hline Smoking causes CHD & 54.2 & 581 & 59.2 & 101 & 36.3 & 1,737 & $40.2^{*}$ & 2419 \\
\hline Smoking causes stroke & 35.4 & 401 & 37.5 & 60 & 16.0 & 791 & $20.1^{*}$ & 1252 \\
\hline Smoking causes impotence & 29.2 & 320 & 29.3 & 46 & 16.6 & 814 & $19.2^{*}$ & 1180 \\
\hline Knowledge of all health effects & 13.2 & 151 & 16.3 & 26 & 5.2 & 261 & $7.0^{*}$ & 438 \\
\hline
\end{tabular}

*Differences between never, former and current smokers significant at $p<001$. 
Table 3 Association between covariates/selected health beliefs and intention to quit among current smokers $(n=4673)$

\begin{tabular}{|c|c|c|c|}
\hline Health beliefs & & $\%$ & OR $(95 \% \mathrm{CI})$ \\
\hline \multirow{4}{*}{$\begin{array}{l}\text { Smoke is dangerous to } \\
\text { non-smokers }\end{array}$} & Disagree & 4.6 & 1.00 \\
\hline & Neither & 4.1 & 0.41 (0.16 to 1.07$)$ \\
\hline & Agree & 88.5 & 1.82 (1.24 to 3.21$)$ \\
\hline & Don't know & 2.9 & $0.37(0.16,0.83)$ \\
\hline \multirow[t]{4}{*}{ Tobacco is addictive } & Disagree & 9.1 & 1.00 \\
\hline & Neither & 5.2 & $0.63(0.39$ to 1.00$)$ \\
\hline & Agree & 83.4 & $1.26(0.90$ to 1.76$)$ \\
\hline & Don't know & 2.4 & $0.91(0.38,2.19)$ \\
\hline \multirow[t]{4}{*}{ Every cigarette damages health } & Disagree & 10.6 & 1.00 \\
\hline & Neither & 10.3 & $1.14(0.70$ to 1.84$)$ \\
\hline & Agree & 75.4 & 5.11 (3.32 to 7.86$)$ \\
\hline & Don't know & 3.7 & $1.64(0.67$ to 4.00$)$ \\
\hline \multirow{4}{*}{$\begin{array}{l}\text { Worried about future damage } \\
\text { from smoking }\end{array}$} & Not at all & 33.7 & 1.00 \\
\hline & A little & 41.7 & 4.01 (3.02 to 5.33$)$ \\
\hline & Very much & 18.9 & 10.44 (7.59 to 14.38$)$ \\
\hline & Don't know & 5.7 & 1.75 (0.99 to 3.09$)$ \\
\hline \multirow[t]{4}{*}{ Smoking has damaged health } & Not at all & 33.2 & 1.00 \\
\hline & A little & 40 & $2.71(2.19$ to 3.36$)$ \\
\hline & Very much & 17.5 & $5.70(4.35$ to 7.47$)$ \\
\hline & Don't know & 9.4 & $1.13(0.69$ to 1.85$)$ \\
\hline \multirow{4}{*}{ How much benefit if quit smoking } & Not at all & 19.3 & 1.00 \\
\hline & A little & 32.1 & 3.50 (2.36 to 5.20$)$ \\
\hline & Very much & 35.2 & 9.96 (6.64 to 14.94$)$ \\
\hline & Don't know & 13.4 & 1.11 (0.70 to 1.77$)$ \\
\hline \multirow[t]{3}{*}{$\begin{array}{l}\text { Knowledge of health effects scale } \\
(0-8)\end{array}$} & $\begin{array}{l}\text { Mean among } \\
\text { those NOT } \\
\text { intending to quit }\end{array}$ & 3.53 & \\
\hline & $\begin{array}{l}\text { Mean among } \\
\text { those intending } \\
\text { to quit }\end{array}$ & 4.71 & \\
\hline & $\begin{array}{l}\text { OR for } 1 \text { unit } \\
\text { increase in score }\end{array}$ & & 1.27 (1.21 to 1.34$)$ \\
\hline
\end{tabular}

$\mathrm{OR}$, 'unadjusted' odds ratio of intending to quit smoking ( 0 , not planning to quit/don't know; 1 , within the next month/within the next 6 months/sometime in the future, beyond 6 months).

differences were observed among sex, income and health knowledge levels.

\section{Other health beliefs}

Current smokers were asked to report additional health beliefs (see table 3). The vast majority of current smokers agreed that smoke is dangerous to non-smokers (88.5\%) and tobacco is addictive (83.4\%). About three-quarters of current smokers agreed that every cigarette damages health. In contrast, less than one-fifth of smokers agreed that smoking has damaged his/her health, one-third of current smokers (33.7\%) reported no worry about the future damage from smoking and approximately $35 \%$ of current smokers agreed that that they would experience future health benefits very much if they quit smoking within the next 6 months.

\section{Predictors of health belief perception among current smokers}

Analyses were conducted to examine the extent to which health knowledge and other health beliefs among current smokers predicted intention to quit. Overall, $31.9 \%$ of current smokers intended to quit at some point in the future. Most of the selected health beliefs were significantly associated with intentions to quit in bivariate analyses, except the belief that 'tobacco is addictive' (see table 3 ).

We conducted multivariate analyses to examine the predictive relation of health knowledge and health beliefs on intention to quit in greater detail. Measures of health beliefs and the health knowledge scale were entered in a logistic regression model predicting intention to quit, adjusting for sex, age, income and education (see table 4). The health knowledge scale, and the health beliefs of 'smoke is dangerous to non-smokers; tobacco is addictive' were not significantly associated with intentions to quit; however, all other health beliefs significantly predicted intentions to quit among current smokers.

\section{DISCUSSION}

The findings of this study shed light onto beliefs about smoking in China-one of the leading public health threats to the country. The results indicate reasonable levels of health knowledge for health effects such as lung cancer, comparable with levels in Western countries. ${ }^{16}$ However, the findings reveal major gaps in the knowledge of other health effects. For example, approximately $40 \%$ of respondents endorsed smoking as a risk factor for heart disease and only one-fifth acknowledged smoking causes stroke-both leading causes of death in China. ${ }^{12}$ In addition, less than two-thirds of respondents agreed that secondhand smoke can cause lung cancer.

In terms of health knowledge towards smoking among different smoking status groups, the findings indicate that Chinese smokers were less likely to agree to health effects than either never smokers or former smokers. For example, over $90 \%$ of never and former smokers agreed that smoking causes lung cancer, compared to only two-thirds of smokers. Similarly, about $83 \%$ of never smokers and $77 \%$ of former smokers agreed that secondhand smoke causes lung cancer compared to only half of smokers. These differences persisted even after adjusting for sociodemographic factors, which suggests that the lower levels of health beliefs among Chinese smokers may reflect either an optimistic bias, lower exposure to health information or both. This finding is similar to patterns observed in Western countries, where smokers systematically underestimate their personal risk from smoking, presumably in attempt to minimise cognitive dissonance from smoking and shield themselves from worry. ${ }^{17-19}$

Compared to historical estimates, the findings suggest that levels of health knowledge about the dangers of smoking may be increasing in China. In particular, data from two decades ago shows that only $51 \%$ of smokers know smoking causes lung cancer, ${ }^{10}$ although differences in the sample profiles complicate comparisons between studies. In addition, the current study only included respondents living in highly developed, urban areas. One might expect levels of health knowledge to be lower in rural areas of China, where access to health information is typically lower. More generally, levels of health knowledge among Chinese smokers were considerably lower than levels previously reported in Western countries. ${ }^{16}$ For example, in Canada and Australia-countries widely acknowledged to be leaders in tobacco control-approximately $90 \%$ of smokers agreed that smoking causes heart disease and $80 \%$ agreed that smoking causes stroke. ${ }^{16}$ In contrast, only $36 \%$ and $16 \%$ of Chinese smokers in the current study agreed that smoking causes heart disease and stroke, respectively. The knowledge awareness gap also exists in secondhand smoke. In Canada and Australia, over $70 \%$ of smokers agreed cigarette smoking causes lung cancer in non-smokers, while only about half of Chinese smokers agree with this statement in current study.

The findings of this study indicate that relatively few Chinese smokers intend to quit smoking. Less than a third of smokers indicated that they intend to quit at any point in the future-levels far below Western countries such as Canada and Australia, where approximately three-quarters of all smokers 
Table 4 Adjusted odds ratios $†$ from logistic regression of selected health beliefs on intention to quit $(n=4673)$

\begin{tabular}{|c|c|c|}
\hline \multicolumn{2}{|l|}{ Covariate } & \multirow{2}{*}{$\begin{array}{l}\mathrm{OR}(95 \% \mathrm{CI}) \\
1.00\end{array}$} \\
\hline Sex & Male & \\
\hline & Female & 1.07 (0.58 to 1.95$)$ \\
\hline \multirow[t]{4}{*}{ Age } & $18-24$ & 1.00 \\
\hline & $25-39$ & $1.02(0.45$ to 2.32$)$ \\
\hline & $40-54$ & 1.28 (0.50 to 3.27$)$ \\
\hline & $55+$ & $1.23(0.51$ to 2.97$)$ \\
\hline \multirow[t]{4}{*}{ Income } & Low & 1.00 \\
\hline & Moderate & $1.09(0.87$ to 1.37$)$ \\
\hline & High & $1.36(0.77$ to 2.41$)$ \\
\hline & No answer & $0.91(0.57$ to 1.46$)$ \\
\hline \multirow[t]{3}{*}{ Education } & Low & 1.00 \\
\hline & Moderate & $0.91(0.67$ to 1.24$)$ \\
\hline & High & 0.92 (0.66 to 1.28$)$ \\
\hline \multirow[t]{4}{*}{ Cigarettes smoked/day } & $0-10$ & 1.00 \\
\hline & $11-20$ & $0.68(0.57$ to 0.81$)$ \\
\hline & $21-30$ & $0.49(0.35$ to 0.68$)$ \\
\hline & $31+$ & $0.48(0.33$ to 0.70$)$ \\
\hline $\begin{array}{l}\text { Knowledge of health effects scale } \\
\text { (OR for } 1 \text { unit increase) }\end{array}$ & \multicolumn{2}{|c|}{$1.03(0.98$ to 1.09$)$} \\
\hline \multirow[t]{4}{*}{ Smoke is dangerous to non-smokers } & Disagree & 1.00 \\
\hline & Neither & $0.62(0.29$ to 1.32$)$ \\
\hline & Agree & $0.97(0.59$ to 1.60$)$ \\
\hline & Don't know & $0.52(0.21,1.28)$ \\
\hline \multirow[t]{4}{*}{ Tobacco is addictive } & Disagree & 1.00 \\
\hline & Neither & $1.02(0.57$ to 1.84$)$ \\
\hline & Agree & $1.05(0.67$ to 1.65$)$ \\
\hline & Don't know & $1.50(0.54,4.20)$ \\
\hline \multirow[t]{4}{*}{ Every cigarette damages health ${ }^{* *}$} & Disagree & 1.00 \\
\hline & Neither & $0.98(0.61$ to 1.57$)$ \\
\hline & Agree & 1.65 (1.15 to 2.38$)$ \\
\hline & Don't know & $2.05(1.07,3.93)$ \\
\hline \multirow{4}{*}{$\begin{array}{l}\text { Worried about future damage from } \\
\text { smoking }^{* * *}\end{array}$} & Not at all & 1.00 \\
\hline & A little & $2.13(1.63$ to 2.78$)$ \\
\hline & Very much & $3.70(2.35$ to 5.83$)$ \\
\hline & Don't know & $1.90(1.18,3.08)$ \\
\hline \multirow[t]{4}{*}{ Smoking has damaged health* } & Not at all & 1.00 \\
\hline & A little & $1.18(0.87$ to 1.60$)$ \\
\hline & Very much & $1.48(1.13$ to 1.94$)$ \\
\hline & Don't know & $0.91(0.56,1.47)$ \\
\hline \multirow[t]{4}{*}{ How much benefit if quit smoking ${ }^{* * *}$} & Not at all & 1.00 \\
\hline & A little & $2.00(1.40$ to 2.86$)$ \\
\hline & Very much & 3.85 (2.52 to 5.88$)$ \\
\hline & Don't know & $0.91(0.57$ to 1.45$)$ \\
\hline
\end{tabular}

Significant levels are indicated as follows:

${ }^{*} \mathrm{p}<0.05 ;{ }^{* *} \mathrm{p}<0.01 ;{ }^{* * *} \mathrm{p}<0.001$.

†Odds of having intention to quit $(0$, not planning to quit/don't know, 1 , within the next month/within the next 6 months/sometime in the future, beyond 6 months).

intend to quit smoking. ${ }^{14}$ As in other studies, intention to quit was positively associated with health beliefs about smoking. ${ }^{16} 20$ In particular, smokers who reported greater worry about the future health effects of smoking and smokers who reported health benefits from quitting were most likely to intend to quit. The direction of this association is likely to be a reciprocal one: greater health beliefs are likely to increase intentions to quit, just as greater intentions are also likely to increase perceived risk and agreement with health effects.

Surveys capable of monitoring perceptions of risk and health knowledge among smokers are not well established globally and they are particular rare among low and middle income countries. ${ }^{1}$ What few data exist from low and middle-income countries, suggest that health knowledge is considerably lower than in high-income countries, consistent with the current findings from China. ${ }^{21-24}$ However, more direct comparisons across studies are precluded by different sample profiles and the use different measures to asses health knowledge.

\section{LIMITATIONS}

The limitations of this study are common to population-based surveys, including potential biases from non-response. For example, in this study, respondents were usually interviewed at night which resulted in lower proportion of young people, especially in the age group 18-24, in the sample than in the general population. The findings should also be interpreted within the context of the sampling frame: only smokers in large urban areas were sampled in this study and one would expect different results in rural areas. It should also be noted that the measures of health knowledge used in this study-agreement with a list of health effects-represents a fairly low threshold for measuring health knowledge. For example, one might expect lower levels with the use of unprompted questions.

\section{IMPLICATIONS}

China accounts for approximately one-third of the world's smokers and China is one of the biggest cigarette production countries in the world. ${ }^{125}$ As a result, health knowledge about smoking in China represents an important indicator for tobacco control in China and global efforts to reduce the health burden from tobacco use. Whereas research from high-income countries shows increasing public concern about the health effects of smoking, the current findings suggest that China lags in this key outcome. However, China has ratified the Framework Convention on Tobacco Control-the world's first treaty devoted to public health - which includes provisions in key areas of tobacco control, including more prominent health warnings, restrictions on tobacco marketing and more prominent public smoking restrictions. Effective implementation of these policy measures will be critically important to increasing perceptions of risk from smoking and helping China to avert the looming public health crisis from tobacco use.

Funding The ITC China Project was supported by grants from the US National Cance Institute (R01 CA125116 and the Roswell Park Transdisciplinary Tobacco Use Research Center (P50 CA111236)), Canadian Institutes of Health Research (79551), Chinese Center for Disease Control and Prevention, and the Ontario Institute for Cancer Research. Additional support was provided by the Propel Centre for Population Health Impact with funds from the Canadian Cancer Society of the National Cancer Institute of Canada/Canadian Cancer Society. The funding sources had no role in the study design, in collection, analysis, and interpretation of data, in the writing of the report, and in the decision to submit the paper for publication.

\section{Competing interests None.}

Patient consent Obtained.

Ethics approval All materials and procedures used in the ITC China Survey were reviewed and cleared for ethics by the Research Ethics Board at the University of Waterloo and by the Institutional Review Board at China National Center for Disease Control and Prevention.

Contributors All of the authors contributed in each of the following ways: conception and design, acquisition of data or analysis and interpretation of data; drafting the article or revising it critically for important intellectual content; final approval of the version published.

Provenance and peer review Not commissioned; externally peer reviewed.

\section{REFERENCES}

1. WHO Report on the global tobacco epidemic, 2008: the MPOWER package. Geneva: World Health Organization, 2008.

2. Yang G, Ma J, Liu N, et al. Smoking and passive smoking in Chinese, 2002. Zhonghua Liu Xing Bing Xue Za Zhi 2005;26:77-83.

3. Yang G, Fan L, Tan J. Smoking in China: findings of the 1996 National Prevalence Survey. JAMA 1999;282:1247-53. 
4. Preventing chronic diseases: a vital investment: WHO Global Report. Geneva: World Health Organization, 2005. http://www.who.int/chp/chronic_disease_report/ full report.pdf

5. Lin $\mathbf{H}$, Murray $\mathrm{M}$, Cohen $\mathrm{T}$, et al. Effects of smoking and solid-fuel use on COPD, lung cancer, and tuberculosis in China: time-based, multiple risk factor, modelling study. Lancet 2008;372:1473-83.

6. Li L, Yong HH, Borland R, et al. Reported awareness of tobacco advertising and promotion in China compared to Thailand, Australia and the USA Tob Control 2009:18:222-7. Published online First: 29 Mar 2009. doi:10.1136/tc.2008.027037.

7. Li 0, Hyland A, O'Connor J, et al. Support for smoke free policies among smokers and non-smokers in six cities in China: ITC China Survey. Tob Control 2010;19 (Suppl 1):i40-46.

8. Curry SJ, Grothaus L, McBride C. Reasons for quitting: intrinsic and extrinsicmotivation for smoking cessation in a population-based sample of smokers. Addict behav 1997;22:727-39.

9. Hyland A, Li Q, Bauer JE, et al. Predictors of cessation in a cohort of current and former smokers followed over 13 years. Nicotine Tob Res 2004;6(suppl 3):S363-69.

10. Wang W, Annette JD. Cigarette smoking and sick leave in an industrial population in Shanghai, China Int J of Epidemiol 1992:21:293-7.

11. Jiang Y, Ong MK, Tong EK, et al. Chinese physicians and their smoking knowledge, attitudes, and practices. AJPM 2007;33:15-22.

12. He J, Gu D, Wu X, et al. Major causes of death among men and women in China. N Engl J Med 2005;353:1124-34

13. Yan J, Xiao S, Ouyang D, et al. Smoking behavior, knowledge, attitudes and practice among health care providers in Changsha city, China. Nicotine Tob Res 2008; 10:737-44.
14. Chan SSC, Sarna L, Wong DCN. Nurses' tobacco-related knowledge, attitudes, and practice in four major cities in China. JNS 2007;39:46-53.

15. Wave 1(2006) ITC China technical report. http://www.itcproject.org/library/countries/ itcchina/reports/technical/finalitcch.

16. Hammond D, Fong GT, McNeill A, et al. Effectiveness of cigarette warning labels in informing smokers about the risks of smoking: findings from the International Tobacco Control (ITC) Four Country Survey. Tob Control 2006;15(Suppl III):iii19-25.

17. Weinstein ND. Accuracy of smokers' risk perceptions. Ann Beh Med 1998;20:135-40.

18. Slovic P. Smoking: risk, perception \& policy. Thousand Oaks, CA: Sage, 2001

19. Weinstein N, Slovic P, Waters E, et al. Public understanding of the illnesses caused by smoking. Nicotine Tob Res 2004;6:349-55.

20. Romer D, Jamieson P. The role of perceived risk in starting and stopping smoking. In: Slovic, ed. Smoking: risk, perception, and policy. Thousand Oaks California: Sage, 2001

21. Unsal M, Hamzacebi $H$, Dabak $S$, et al. Smoking status and levels of knowledge regarding cigarettes among primary school teachers. South Med $J$ 2008;101:1227-31.

22. Singh V, Gupta R. Prevalence of tobacco use and awareness of risks among schoo children in Jaipur. J Assoc Physicians India 2006;54:609-12.

23. Al-Haddad NM, Hamadeh RR, Bahram SA. Public knowledge and attitudes towards passive smoking. Saudi Med J 2005;26:2004-6.

24. Khan JA, Hussain SF, Malik A, et al. Knowledge, attitudes and the prevalence of smoking among hospital attendants in a developing country. Trop Doct 2003;33:231-4.

25. The World Market for Tobacco. Euromonitor International 2007. http://www. euromonitor.com/The_World_Market_for_Tobacco (accessed 4 Apr 2008) 